Hans P. Heinig and Adrian L. Lee, Department of Mathematics and Statistics, McMaster University, Hamilton, Ontario, Canada, L8S 4K1

\title{
SHARP PALEY-TITCHMARSH INEQUALITIES IN ORLICZ SPACES
}

\author{
Abstract \\ Let $(T f)(x)=x \hat{f}(x)$, where $\hat{f}$ is the Fourier transform of $f$. If \\ $P(t)=t \int_{0}^{t} s^{-2} Q(s) d s, t>0$, where $Q$ is some non-negative continuous \\ function, then there is a constant $A>0$, such that

$$
\int_{\mathbb{R}} Q(|T f(x)|) \frac{d x}{x^{2}} \leq A \int_{\mathbb{R}} P(|f(x)|) d x
$$ \\ holds. Moreover, if this inequality is satisfied for all $f$, then $t \int_{0}^{t} s^{-2} Q(s) d s$ \\ $\leq C P(t), t>0$, for some constant $C>0$. \\ Corresponding Orlicz space and dual inequalities for this and the \\ Hardy averaging operator are also given.
}

\section{Introduction}

The classical Paley-Titchmarsh inequality asserts that if $f \in L^{p}, 1<p<2$, then

$$
\int_{-\infty}^{\infty}|x|^{p-2}|\hat{f}(x)|^{p} d x \leq C \int_{-\infty}^{\infty}|f(x)|^{p} d x
$$

where $\hat{f}$ is the Fourier transform of $f$ defined by

$$
\hat{f}(x)=\int_{-\infty}^{\infty} e^{-i x t} f(t) d t, \quad x \in \mathbb{R} .
$$

Key Words: Orlicz spaces, modular inequalities, Fourier transform

Mathematical Reviews subject classification: Primary: 42A38, 46E30; Secondary: $26 \mathrm{D} 15$

Received by the editors February 8, 1995

* Research supported in part by NSERC grant A-4837 
The object of this note is to extend this inequality to the form

$$
\int_{-\infty}^{\infty} Q(|x \hat{f}(x)|) \frac{d x}{x^{2}} \leq A \int_{-\infty}^{\infty} P^{*}(|f(x)|) d x,
$$

where $P^{*}$ and $Q$ are certain non-negative continuous, non-decreasing functions. Moreover, it is shown (Theorem 2.1) that the $P^{*}$ and $Q$ for which (1.2) is satisfied are in some sense the best possible. Since (1.1) fails where $p=1$, one might also expect that $Q$ and $P^{*}$ are convex. However this need not be the case (cf. Ex. 2.1). Since (1.2) is proved via an interpolation argument one might expect that sharp estimates of the form (1.2) can be obtained for operators other than those of Fourier type. This is indeed the case, and we illustrate this for the Hardy averaging operator.

Although modular inequalities such as (1.2) imply corresponding Orlicz space inequalities, we show also in Section 2 that these inequalities are sharp.

Typically $L^{p}$-inequalities imply dual inequalities, so specifically the dual estimate of (1.1) is

$$
\int_{-\infty}^{\infty}|\hat{f}(x)|^{q} d x \leq C \int_{-\infty}^{\infty}|x|^{q-2}|f(x)|^{q} d x, \quad q>2 .
$$

In Section 3 we obtain dual inequalities of (1.2) which are established by modifying recent results of S. Bloom and R. Kerman [1]. Since these estimates are in terms of complementary functions we require that $Q$ be a Young's function.

If $\Phi$ is a non-negative increasing function on $\mathbb{R}^{+}=[0, \infty)$, then a $\mu$-measurable function $f$ on $X$ belongs to the Orlicz space $L_{\Phi(\mu)}$, if

$$
\|f\|_{\Phi(\mu)}=\inf \left\{\lambda>0: \int_{X} \Phi\left(\frac{|f(x)|}{\lambda}\right) d \mu(x) \leq 1\right\}
$$

is finite. If $d \mu(x)=u(x) d x, u$ a non-negative weight function then we write $\|f\|_{\Phi(u)}$ or, if $u \equiv 1,\|f\|_{\Phi}$.

Constants are denoted by $A, B, C, D$ and may be different at different places, while $p^{\prime}$ and $q^{\prime}$ are as usual the conjugate indices of $p$ and $q$, respectively. Finally $\chi_{E}$ denotes the characteristic function of the set $E$.

Other notations are given as they arise and we refer to [5] and [6] for additional notation and elementary properties used.

\section{Sharp Inequalities}

The following known interpolation result is an extension of a result of A. Zygmund [8]. See also S. Koizumi [3] where a similar result is given. 
Theorem 2.1 ([2, Theorem 2$)]$ Let $T$ be a quasi-linear operator defined for simple functions on $(X, \mathcal{M}, \mu)$ with values in the space of functions on $(Y, \mathcal{N}, \nu)$. Suppose $T$ is of weak type $(a, a)$ and $(b, b), 1 \leq a<b<\infty$ and $Q$ a non-negative continuous, non-decreasing function, such that $Q(0)=0$ and $Q(2 x)=O(Q(x)), x \rightarrow \infty$. If

$$
P(x)=x^{a} \int_{0}^{x} t^{-1-a} Q(t) d t
$$

and

$$
\begin{aligned}
\int_{x}^{\infty} t^{-b-1} P(t) d t & =O\left(x^{-b} P(x)\right), x \rightarrow \infty \\
\int_{x}^{1} t^{-b-1} P(t) d t & =O\left(x^{-b} P(x)\right), x \rightarrow 0
\end{aligned}
$$

then

$$
\int_{Y} Q(|T f|) d v \leq C \int_{X} P(|f|) d \mu .
$$

In particular $T$ can be extended uniquely to the space of all $P(|f|)-\mu$ integrable functions preserving (2.3).

If $T$ is defined by $(T f)(x)=x \hat{f}(x), x \in \mathbb{R}$, then it is well known (c.f. [7]) that $T$ is of weak type $(1,1)$ and strong (hence weak) type $(2,2)$ with respect to the measure

$$
\mu(E)=\int_{E} x^{-2} d x, \quad E \subset \mathbb{R} \backslash\{0\} .
$$

It is now easy to prove the following result:

Theorem 2.2 Suppose $Q$ satisfies the hypotheses of Theorem 2.1. If $P$ is defined by (2.1) with $a=1, b=2$ and satisfies (2.2), then (1.2) holds for some constant $A>0$, if and only if, $P(x) \leq B P^{*}(x)$ for some constant $B>0$.

Proof. By Theorem 2.1 with $a=1, b=2$ and $X=Y=\mathbb{R}$

$$
\int_{\mathbb{R}} Q(|x \hat{f}(x)|) \frac{d x}{x^{2}} \leq C \int_{\mathbb{R}} P(|f(x)|) d x \leq C B \int_{\mathbb{R}} P^{*}(|f(x)|) d x,
$$

so (1.2) holds with $A=C B$. 
Conversely, if (1.2) holds for all $f$, then with $f(x)=r^{-1} \chi_{(0, r)}(|x|), r>0$ fixed, (1.2) yields

$$
\begin{aligned}
2 A r P^{*} & \left(\frac{1}{r}\right)=A \int_{-r}^{r} P^{*}\left(\frac{1}{r}\right) d x=A \int_{\mathbb{R}} P^{*}(|f(x)|) d x \\
& \geq \int_{0}^{\infty} Q\left(\left|x \int_{0}^{r} \frac{\cos x t}{r} d t\right|\right) \frac{d x}{x^{2}} \geq \int_{0}^{1 / r} Q\left(\frac{x}{r} \int_{0}^{r} \cos x t d t\right) \frac{d x}{x^{2}} \\
& \geq \int_{0}^{1 / r} Q(x \cos 1) \frac{d x}{x^{2}} \geq \frac{1}{D} \int_{0}^{1 / r} Q(x) \frac{d x}{x^{2}},
\end{aligned}
$$

where the last inequality follows from the fact that $Q(2 x) \leq D Q(x)$. Writing $x=1 / r$, the result follows with $B=2 A D$.

Remark 2.3 The higher dimensional analogue of the Paley-Titchmarsh inequality (1.2) is

$$
\int_{\mathbb{R}^{n}} Q\left(|x|^{n}|\hat{f}(x)|\right) \frac{d x}{|x|^{2 n}} \leq A \int_{\mathbb{R}^{n}} P^{*}(|f(x)|) d x .
$$

If $P$ and $Q$ are as in Theorem 2.2, then this inequality holds if and only if $P(x) \leq B P^{*}(x)$. The sufficiency is proved with the obvious modifications again via Theorem 2.1. To prove necessity one defines the test function by $f(x)=r^{-n} \chi_{(0, r)}(|x|), x \in \mathbb{R}^{n}, r>0$. This is radial and since the Fourier transform of a radial function $f$ is radial and given by

$$
(2 \pi)^{1-n / 2} t^{1-n / 2} \int_{0}^{\infty} s^{n / 2} J_{(n-2) / 2}(t s) f(s) d s,
$$

where

$$
J_{(n-2) / 2}(t s)=\frac{2^{-n / 2} \pi^{-1 / 2}(t s)^{(n-2) / 2}}{\Gamma\left(\frac{n}{2}-\frac{1}{2}\right)} \int_{0}^{\pi / 2} \cos (t s \cos y) \sin ^{n-2} y d y
$$

is the Bessel function. On estimating this from below and writing $x=r^{-n}$, one obtains the result (c.f. [6], where such detailed estimates are given).

Besides the Fourier type operator considered in Theorem 2.2 one also obtains such modular inequalities as (1.2) for other classical operators such as the Hardy-Littlewood maximal operator or the Hardy averaging operator

$$
(T f)(x)=x^{-1} \int_{0}^{x} f(t) d t, \quad x>0 .
$$

For this operator we have the following result: 
Proposition 2.4 Suppose $P$ and $Q$ satisfy the conditions of Theorem 2.2. Then the inequality

$$
\int_{0}^{\infty} Q\left(\left|x^{-1} \int_{0}^{x} f\right|\right) d x \leq A \int_{0}^{\infty} P^{*}(|f|) d x
$$

holds, if and only if there is a constant $B>0$, such that $P(x) \leq B P^{*}(x)$, $x>0$.

Proof. Since $T$ given by $(2.5)$ is of weak type $(1,1)$ and strong type $(2,2)$ (in fact $(p, p), 1<p<\infty),(2.6)$ follows from Theorem 2.1.

Conversely, if $f(x)=r^{-1} \chi_{(0, r)}(x), r>0$, in (2.6), then

$$
A r P^{*}(1 / r) \geq \int_{r}^{\infty} Q\left(x^{-1} \int_{0}^{r} r^{-1} d t\right) d x=\int_{r}^{\infty} Q(1 / x) d x=\int_{0}^{1 / r} Q(s) s^{-2} d s
$$

and the result follows with $x=1 / r$ and $B=A$.

Example 2.5 a) Let $1<p<q<2$ and define $Q$ by

$$
Q(x)= \begin{cases}x^{q} / q^{\prime} & \text { if } 0<x \leq 1 \\ 1 / p-1 / q+x^{p} / p^{\prime} & \text { if } \quad x>1\end{cases}
$$

Since $P(t)=t \int_{0}^{t} s^{-2} Q(s) d s$, an easy calculation shows that

$$
P(t)= \begin{cases}t^{q} / q & \text { if } 0<t \leq 1 \\ 1 / q-1 / p+t^{p} / p & \text { if } t>1\end{cases}
$$

Clearly $P$ and $Q$ satisfy the conditions of Theorem 2.2 and hence (1.2) holds in this case.

b) If $1<p<2$ and $0 \leq \alpha$ define $Q$ by

$$
Q(x)= \begin{cases}x^{p} / p^{\prime} & \text { if } 0<x \leq 1 \\ x-1 / p & \text { if } x>1 \text { and } \alpha=0 \\ 1 / p^{\prime}+x(\ln x)^{\alpha} & \text { if } x>1 \text { and } \alpha>0\end{cases}
$$

then

$$
P(t)= \begin{cases}t^{p} / p & \text { if } 0<x \leq 1 \\ 1 / p+t \ln t & \text { if } t>1 \text { and } \alpha=0 \\ t+t(\ln t)^{\alpha+1} /(\alpha+1)-1 / p^{\prime} & \text { if } t>1 \text { and } \alpha>0 .\end{cases}
$$

Since an easy calculation shows that $P$ and $Q$ satisfy the conditions of Theorem 2.2, both (1.2) and (2.6) hold in this case. 
Note that if $\alpha=0, p=2$, the function $P$ does not satisfy conditions (2.2). Yet it follows from a recent result of $S$. Koizumi $[4, \S 2]$ that a Paley-Titchmarsh inequality of the form (1.2) with

$$
P^{*}(t)= \begin{cases}t^{2} / 2 & \text { if } 0<t \leq 1 \\ t / 2(1+\ln t) & \text { if } t>1\end{cases}
$$

holds. However, since the operator $T f(x)=x \hat{f}(x)$ is of strong type $(2,2)$ and Theorem 2.1 requires only that $T$ is of weak type $(2,2)$, this result is not unexpected.

Observe also that $Q(x)$ for $1<x<e^{1-\alpha}, 0<\alpha<1$ is not convex.

Denote by $T_{1}$ and $T_{2}$ the operators

$$
T_{1} f(x)=x \hat{f}(x), x \in \mathbb{R}, \quad T_{2} f(x)=x^{-1} \int_{0}^{x} f, x>0,
$$

then we have the following Orlicz space estimates:

Theorem 2.6 Suppose $P$ and $Q$ are as in Theorem 2.1 and $\mu$ is defined by (2.4). If $P^{*}$ is convex and satisfies $P^{*}(2 x) \leq C P^{*}(x)$, then

$$
\left\|T_{1} f\right\|_{Q(\mu)} \leq A\|f\|_{P^{*}} \quad \text { and } \quad\left\|T_{2} f\right\|_{Q} \leq A\|f\|_{P^{*}},
$$

if and only if there is a constant $B>0$, such that $P(x) \leq B P^{*}(x)$.

Proof. Consider $T_{1}$ first. By Theorem 2.2

$$
\begin{aligned}
\left\|T_{1} f\right\|_{Q(\mu)} & =\inf \left\{\lambda>0: \int_{\mathbb{R}} Q\left(\frac{\left|\left(T_{1} f\right)(x)\right|}{\lambda}\right) \frac{d x}{x^{2}} \leq 1\right\} \\
& \leq \inf \left\{\lambda>0: A \int_{\mathbb{R}} P^{*}\left(\frac{|f(x)|}{\lambda}\right) d x \leq 1\right\} .
\end{aligned}
$$

But $P^{*}$ is convex, hence (w.l.g. take $A>1$ ), $A P^{*}(t / A) \leq P^{*}(t)$, so that

$$
\left\|T_{1} f\right\|_{Q(\mu)} \leq \inf \left\{A(\lambda / A)>0: \int_{\mathbb{R}} P^{*}\left(\frac{A|f(x)|}{\lambda}\right) d x \leq 1\right\}=A\|f\|_{P^{*}} .
$$

Conversely, if (2.7) holds for all $f$, then specifically with $f(x)=$ $r^{-1} \chi_{(0, r)}(|x|), r>0$ 


$$
\begin{aligned}
A & \frac{1}{r\left(P^{*}\right)^{-1}\left(\frac{1}{2 r}\right)} \\
& =A \inf \left\{\lambda>0: P^{*}\left(\frac{1}{\lambda r}\right) \leq \frac{1}{2 r}\right\}=A \inf \left\{\lambda>0: \int_{-r}^{r} P^{*}\left(\frac{1}{\lambda r}\right) d x \leq 1\right\} \\
& =A\|f\|_{P^{*}} \\
& \geq\left\|T_{1} f\right\|_{Q(\mu)} \geq \inf \left\{\lambda>0: \int_{0}^{1 / r} Q\left(\frac{x}{\lambda r} \int_{0}^{r} \cos x t d t\right) \frac{d x}{x^{2}} \leq 1\right\} \\
& \geq \inf \left\{\lambda>0: \int_{0}^{1 / r} Q\left(\frac{x \cos 1}{\lambda}\right) \frac{d x}{x^{2}} \leq 1\right\} \quad(x \cos 1=\lambda t) \\
& =\inf \left\{\lambda>0: \int_{0}^{\cos 1 /(r \lambda)} Q(t) \frac{\lambda}{\cos 1} \frac{(\cos 1)^{2}}{\lambda^{2} t^{2}} d t \leq 1\right\} \\
& =\inf \left\{\lambda>0: \frac{\cos 1}{\lambda} \frac{P\left(\frac{\cos 1}{r \lambda}\right)}{\frac{\cos 1}{r \lambda}} \leq 1\right\}=\inf \left\{\lambda>0: r P\left(\frac{\cos 1}{r \lambda}\right) \leq 1\right\} \\
& =\frac{\cos 1}{r P^{-1}\left(\frac{1}{r}\right)} \cdot
\end{aligned}
$$

Since $\left(P^{*}\right)^{-1}$ is concave this yields

$$
\frac{1}{2}\left(P^{*}\right)^{-1}\left(\frac{1}{r}\right) \leq\left(P^{*}\right)^{-1}\left(\frac{1}{2 r}\right) \leq \frac{A}{\cos 1} P^{-1}\left(\frac{1}{r}\right)
$$

and with $x=P^{-1}\left(\frac{1}{r}\right)$ we get $\left(P^{*}\right)^{-1}(P(x)) \leq \frac{2 A}{\cos 1} x$, or

$$
P(x) \leq P^{*}\left(\frac{2 A}{\cos 1} x\right) \leq D P^{*}(x) .
$$

To prove the result for $T_{2}$, the sufficiency part follows from Proposition 2.4, and the necessity part is obtained by considering the test function $f(x)=$ $r^{-1} \chi_{(0, r)}(x), r>0, x>0$. We omit the details.

\section{Dual Estimates}

Recall ([5]) that a function $P$ defined by

$$
P(x)=\int_{0}^{x} p(t) d t, \quad x>0,
$$


where $p$ is non-decreasing, right continuous on $(0, \infty)$ and $p(0)=0$, is called a Young's function. If $p^{-1}$ is the right continuous inverse of $p$, then the complementary Young's function of $P$ is

$$
\tilde{P}(x)=\int_{0}^{x} p^{-1}(t) d t
$$

or equivalently $\tilde{P}(x)=\sup _{t \geq 0}[x t-P(t)]$.

The following two results are extensions of results of S. Bloom and R. Kerman [1, Prop. 2.5 and Cor. 2.7]:

Proposition 3.1 Suppose $(X, \mathcal{M}, \mu)$ and $(Y, \mathcal{N}, \nu)$ are $\sigma$-finite measure spaces. Let $T$ be an operator mapping functions $f$ on $X$ to $T f$ on $Y$. If $P$ and $Q$ are Young's functions, then

$$
\int_{Y} Q(|T f(y)|) d \nu(y) \leq \int_{X} P(C|f(x)|) d \mu(x)
$$

is satisfied for all $f$, if and only if there is a $C>0$, such that for every $\varepsilon>0$

$$
\|T f\|_{Q(\varepsilon d \nu)} \leq C\|f\|_{P(\varepsilon d \mu)}
$$

holds. Here $C>0$ is independent of $\varepsilon$.

Proof. The proof is essentially that of [1, Prop. 2.5], where the case $P=Q$ an $\mathrm{N}$-function was proved. We give a proof here for completeness only.

Suppose (3.2) holds and let $\varepsilon=\left(\int_{X} P(|f|) d \mu\right)^{-1}$. Then

$$
\begin{aligned}
\|f\|_{P(\varepsilon d \mu)} & =\inf \left\{\lambda>0: \int_{X} P\left(\frac{|f|}{\lambda}\right) \varepsilon d \mu \leq 1\right\} \\
& =\inf \left\{\lambda>0: \int_{X} P\left(\frac{|f|}{\lambda}\right) d \mu \leq \int_{X} P(|f|) d \mu\right\}=1
\end{aligned}
$$

and hence $\|T f\|_{Q(\varepsilon d \nu)} \leq C$. The homogeneity of the norm and the linearity of $T$ then imply that $\|T(f / C)\|_{Q(\varepsilon d \nu)} \leq 1$. Therefore

$$
\|T(f / C)\|_{Q(\varepsilon d \nu)}=\inf \left\{\lambda>0: \int_{Y} Q\left(\frac{|T(f / C)|}{\lambda}\right) \varepsilon d \nu \leq 1\right\} \leq 1 .
$$

and hence

$$
\int_{Y} Q(|T(f / C)|) d \nu \leq \frac{1}{\varepsilon}=\int_{X} P(|f|) d \mu .
$$


(3.1) follows then on replacing $f / C$ by $f$.

Conversely, if (3.1) is satisfied, fix $f$ and let $\alpha=\|f\|_{P(\varepsilon d \mu)}$. Then

$$
1=\|f / \alpha\|_{P(\varepsilon d \mu)}=\inf \left\{\lambda>0: \int_{X} P\left(\frac{|f|}{\lambda \alpha}\right) \varepsilon d \mu \leq 1\right\},
$$

so that

$$
\int_{X} P\left(\frac{|f|}{\alpha}\right) \varepsilon d \mu \leq 1
$$

But by (3.1)

$$
\int_{Y} Q\left(\frac{|T f|}{\alpha C}\right) \varepsilon d \nu=\varepsilon \int_{Y} Q(|T(f / \alpha C)|) d \nu \leq \frac{\varepsilon}{\varepsilon}=1,
$$

so that,

$$
\|T f\|_{Q(\varepsilon d \nu)}=\inf \left\{\lambda>0: \int_{Y} Q\left(\frac{|T f|}{\lambda}\right) \varepsilon d \nu \leq 1\right\} \leq \alpha C=C\|f\|_{P(\varepsilon d \mu)} .
$$

Corollary 3.2 Let $u_{i}, v_{i}, i=0,1$ be non-negative weight functions on $X$, respectively, $Y$. Suppose $(X, \mathcal{M}, \mu),(Y, \mathcal{N}, \nu), T, P$ and $Q$ are as in Proposition 3.1 with $d \mu(x)=u_{0}(x) d x, d \nu(y)=v_{0}(y) d y$ and $\tilde{P}, \tilde{Q}$ are the complementary functions of $P$, respectively, $Q$. If $T^{*}$ is the adjoint of $T$, then

$$
\int_{Y} Q\left(v_{1}(y)|(T f)(y)|\right) v_{0}(y) d y \leq \int_{X} P\left(C u_{1}(x)|f(x)|\right) u_{0}(x) d x
$$

is satisfied, if and only if,

$$
\begin{aligned}
\int_{X} \tilde{P}\left(\left(u_{0}(x) u_{1}(x)\right)^{-1} \mid\left(T^{*} g\right)\right. & (x) \mid) u_{0}(x) d x \\
& \leq \int_{Y} \tilde{Q}\left[C\left(v_{0}(y) v_{1}(y)\right)^{-1}|g(y)|\right] v_{0}(y) d y
\end{aligned}
$$

holds.

Proof. The proof is as in [1, Cor. 2.7] and is given here for completeness only.

Suppose (3.3) holds, let $S f=\left(u_{0} u_{1}\right)^{-1} T^{*}\left(v_{1} v_{2} f\right)$, then (3.4) has the form

$$
\int_{X} \tilde{P}(|S f|) u_{0}(x) d x \leq \int_{Y} \tilde{Q}[C|f|] v_{0}(y) d y .
$$


But if $P$ and $Q$ are Young's functions, so are $\tilde{P}$ and $\tilde{Q}$ and hence by Proposition $3.1,(3.5)$ is equivalent to

$$
\|S f\|_{\tilde{P}\left(\varepsilon u_{0}\right)} \leq C\|f\|_{\tilde{Q}_{\left(\varepsilon v_{0}\right)}}
$$

for every $\varepsilon>0$. By duality and Hölder's inequality in Orlicz spaces (c.f. [5])

$$
\begin{aligned}
\|S f\|_{\tilde{P}\left(\varepsilon u_{0}\right)} & =\sup _{\|f\|_{P\left(\varepsilon u_{0}\right)} \leq 1}\left|\int_{X} S f(x) g(x) \varepsilon u_{0}(x) d x\right| \\
& \leq \sup _{\|g\|_{P\left(\varepsilon u_{0}\right)} \leq 1}\left|\int_{X}\left(u_{0} u_{1}\right)^{-1} T^{*}\left(v_{0} v_{1} f\right) g \varepsilon u_{0} d x\right| \\
& =\sup _{\|g\|_{P\left(\varepsilon u_{0}\right)} \leq 1}\left|\int_{Y} v_{0} v_{1} f T\left(g / u_{1}\right) \varepsilon d y\right| \\
& \leq C \sup _{\|g\|_{P\left(\varepsilon u_{0}\right)} \leq 1}\|f\|_{\tilde{Q}\left(\varepsilon v_{0}\right)}\left\|v_{1} T\left(g / u_{1}\right)\right\|_{Q\left(\varepsilon v_{0}\right)} \\
& \leq C\|f\|_{\tilde{Q}\left(\varepsilon v_{0}\right)},
\end{aligned}
$$

provided $\left\|v_{1} T\left(g / u_{1}\right)\right\|_{Q\left(\varepsilon v_{0}\right)} \leq C\|g\|_{P\left(\varepsilon u_{0}\right)}$. But if we write $T_{1} g=v_{1} T\left(g / u_{1}\right)$, then by Proposition 3.1, this is equivalent to

$$
\int_{Y} Q\left(\left|T_{1} g\right|\right) v_{0} d y \leq \int_{X} P(C|g|) u_{0} d x
$$

or

$$
\int_{Y} Q\left(v_{1}\left|T\left(g / u_{1}\right)\right|\right) v_{0} d y \leq \int_{X} P(C|g|) u_{0} d x
$$

and with $f=g / u_{1}$, this is (3.3).

Conversely, if (3.4) holds, then so does (3.5) which by Proposition 3.1 is equivalent to (3.6).

Now (3.3) with $T_{1} g=v_{1} T g$ has the form

$$
\int_{Y} Q\left(\left|T_{1}\left(f / u_{1}\right)\right|\right) v_{0} d y \leq \int_{X} P(C|f|) u_{0} d x
$$

which by Proposition 3.1 is equivalent to

$$
\left\|T_{1}\left(f / u_{1}\right)\right\|_{Q\left(\varepsilon v_{0}\right)} \leq C\|f\|_{P\left(\varepsilon u_{0}\right)}
$$


for every $\varepsilon>0$. But again, duality and Hölder's inequality yield

$$
\begin{aligned}
\left\|T_{1}\left(f / u_{1}\right)\right\|_{Q\left(\varepsilon v_{0}\right)} & =\sup _{\|h\|_{\tilde{Q}\left(\varepsilon v_{0}\right)} \leq 1}\left|\int_{Y} T_{1}\left(f / u_{1}\right) h \varepsilon v_{0} d y\right| \\
& =\sup _{\|h\|_{\tilde{Q}\left(\varepsilon v_{0}\right)} \leq 1}\left|\int_{Y} v_{1} T\left(f / u_{1}\right) h \varepsilon v_{0} d y\right| \\
& =\sup _{\|h\|_{\tilde{Q}\left(\varepsilon v_{0}\right)} \leq 1}\left|\int_{Y}\left(u_{1} u_{0}\right)^{-1} f T^{*}\left(v_{0} v_{1} h\right) u_{0} \varepsilon d x\right| \\
& \leq C \sup _{\|h\|_{\tilde{Q}\left(\varepsilon v_{0}\right)} \leq 1}\|f\|_{P\left(\varepsilon u_{0}\right)}\left\|\left(u_{0} u_{1}\right)^{-1} T^{*}\left(v_{0} v_{1} h\right)\right\|_{\tilde{P}\left(\varepsilon u_{0}\right)} \\
& \leq C\|f\|_{P\left(\varepsilon u_{0}\right)},
\end{aligned}
$$

since $\left\|\left(u_{0} u_{1}\right)^{-1} T^{*}\left(v_{0} v_{1} h\right)\right\|_{\tilde{P}\left(\varepsilon u_{0}\right)} \leq C\|h\|_{\tilde{Q}\left(\varepsilon v_{0}\right)}$ by Proposition 3.1 and the fact that (3.4) is satisfied.

In order to apply Corollary 3.2 to the operators considered in Section 1, we must ensure that $Q$ and $P$ (or $P^{*}$ ) of Theorem 2.2 are Young's functions.

Let

$$
Q(x)=\int_{0}^{x} q(t) d t, \quad x>0
$$

where $q(0)=0, q$ a right continuous non-decreasing function. Then $Q$ is a Young's function and $\tilde{Q}(x)=\int_{0}^{x} q^{-1}(t) d t, x>0$ is the complementary function of $Q$.

Now if

$$
P(x)=\int_{0}^{x} p(t) d t, \quad x>0
$$

where $p(t)=\int_{0}^{t} \frac{q(s)}{s} d s$, then $P$ is a Young's function and

$$
\begin{aligned}
P(x) & =\int_{0}^{x} \int_{0}^{t} \frac{q(s)}{s} d s d t=\int_{0}^{x} \frac{q(s)}{s}(x-s) d s=x \int_{0}^{x} s^{-1} d Q(s)-Q(x) \\
& =x\left[\left.s^{-1} Q(s)\right|_{0} ^{x}+\int_{0}^{x} s^{-2} Q(s) d s\right]-Q(x) \\
& =x \int_{0}^{x} s^{-2} Q(s) d s .
\end{aligned}
$$

Hence (2.1) holds with $a=1$. Also

$$
\tilde{P}(x)=\int_{0}^{x} p^{-1}(s) d s=\int_{0}^{p^{-1}(x)} q(t) d t=Q\left(p^{-1}(x)\right)
$$


is the complementary function of $P$.

The result dual to Theorem $2.2\left(\right.$ with $\left.P^{*}=P\right)$ is

Proposition 3.3 Suppose $P$ and $Q$ are the Young's functions given above with complementary functions $\tilde{P}$, $\tilde{Q}$. If $Q(2 x)=O(Q(x)), x \rightarrow \infty$, and $P$ satisfies (2.2) with $b=2$, then

$$
\int_{\mathbb{R}} \tilde{P}(|\hat{f}(x)|) d x \leq \int_{\mathbb{R}} \tilde{Q}(C|y f(y)|) \frac{d y}{y^{2}} .
$$

Proof. By Theorem 2.2, (1.2) holds with $P^{*}=P$, and since $P$ is convex and $A \geq 1$ (w.l.g.) it follows that

$$
\int_{\mathbb{R}} Q(|x \hat{f}(x)|) \frac{d x}{x^{2}} \leq \int_{\mathbb{R}} P(A|f(x)|) d x .
$$

Applying Corollary 3.2 with $u_{0}=u_{1}=1, v_{0}(y)=y^{-2}, v_{1}(y)=|y|$, (3.8) follows.

Remark 3.4 Under the same conditions on $P$ and $Q$ one also obtains an estimate for the adjoint Hardy operator:

$$
\int_{0}^{\infty} \tilde{P}\left(\left|\int_{x}^{\infty} \frac{f(t)}{t} d t\right|\right) d x \leq \int_{0}^{\infty} \tilde{Q}(A|f(x)|) d x .
$$

Moreover, it is easily seen that (3.9) also holds for the Hardy operator if one replaces in (2.6) the averaging operator by its adjoint.

Note also, that if $f(x)=x^{-1} \chi_{(r, \infty)}(x), r>0, x>0$ then (3.9) implies that

$$
\int_{r}^{\infty} \tilde{Q}(A / x) d x=A \int_{0}^{A / r} \tilde{Q}(s) s^{-2} d s \geq \int_{0}^{r} \tilde{P}\left(\int_{r}^{\infty} t^{-2} d t\right) d x=r \tilde{P}\left(\frac{1}{r}\right)
$$

and with $t=1 / r, \tilde{P}(t) \leq A t \int_{0}^{A t} s^{-2} \tilde{Q}(s) d s$.

We illustrate (3.8) by an example.

Example 3.5 Let $1<p<2$ and define

$$
Q(x)= \begin{cases}x^{p} / p^{\prime} & \text { if } \quad 0<x \leq 1 \\ (p-1) x \ln x+1 / p^{\prime} & \text { if } \quad x>1\end{cases}
$$


then $Q$ is continuous and $Q(2 x)=O(Q(x))$ as $x \rightarrow \infty$. Since $P(x)=$ $x \int_{0}^{x} t^{-2} Q(t) d t$ an easy calculation shows that

$$
P(x)= \begin{cases}x^{p} / p & \text { if } 0<x \leq 1 \\ x+\frac{x(p-1)}{2}(\ln x)^{2}-1 / p^{\prime} & \text { if } \quad x>1\end{cases}
$$

satisfies (2.2) with $b=2$.

Next, we compute $\tilde{Q}$ and $\tilde{P}$. Since

$$
\begin{gathered}
q(x)= \begin{cases}(p-1) x^{p-1} & \text { if } 0<x \leq 1 \\
(p-1) \ln x+p-1 & \text { if } x>1,\end{cases} \\
q^{-1}(s)= \begin{cases}(s /(p-1))^{1 /(p-1)} & \text { if } 0<s \leq(p-1) \\
e^{s /(p-1)-1} & \text { if } s>p-1,\end{cases} \\
\tilde{Q}(x)= \begin{cases}q^{-1}(s) d s & \text { if } 0<x \leq p-1 \\
= & \text { if } x>p . \\
\frac{(p-1)^{p^{\prime}}}{p} x^{p^{\prime}} & \end{cases}
\end{gathered}
$$

Finally

$$
p(t)=\int_{0}^{t} s^{-1} q(s) d s= \begin{cases}t^{p-1} & \text { if } 0<t \leq 1 \\ 1+\frac{p-1}{2}(\ln t)^{2}+(p-1) \ln t & \text { if } t>1\end{cases}
$$

so that

$$
p^{-1}(x)= \begin{cases}x^{1 /(p-1)} & \text { if } 0<x \leq 1 \\ e^{\sqrt{2(x-1) /(p-1)+1}-1} & \text { if } x>1\end{cases}
$$

But since $\tilde{P}(x)=Q\left(p^{-1}(x)\right)$, it follows that

$$
\tilde{P}(x)= \begin{cases}x^{p^{\prime}} / p & \text { if } 0<x \leq 1 \\ (p-1) e^{-1+\sqrt{2(x-1) /(p-1)+1}} & \\ \times[\sqrt{2(x-1) /(p-1)+1}-1]+\frac{1}{p^{\prime}} & \text { if } x>1 .\end{cases}
$$

Applying Proposition 3.3 it follows that (3.8) holds with $\tilde{P}$ and $\tilde{Q}$ given by (3.11) and (3.10). 


\section{References}

[1] S. Bloom and R. Kerman, Weighted $L^{\phi}$ integral inequalities for operators of Hardy type, Studia Math., 110(1) (1994), 34-52.

[2] H. P. Heinig, On an interpolation theorem of Zygmund and Koizumi, Can. Math. Bull., 13 (1970), 221-226.

[3] S. Koizumi, Contributions to the theory of interpolation of operations, Osaka J. Math., 8 (1971), 135-149; ibid. 10 (1973), 131-145.

[4] S. Koizumi, A remarkable Banach function space on the theory of interpolation and extrapolation of operations, Keio Sci. \& Techn. Reports, 46(2) (1994), 11-20.

[5] M. A. Krasnosel'skii and Ya. B. Rutickii, Convex functions and Orlicz spaces, Noordhoff, Groningen, 1961.

[6] A. L. Lee, The Hausdorff-Young and Paley-Titchmarsh inequalities in Orlicz spaces, M.Sc. Thesis, McMaster University, Hamilton, Canada, Dec. 1994.

[7] E. M. Stein and G. Weiss, Introduction to Fourier analysis on Euclidean spaces, Princeton Univ. Press, N.J., 1971.

[8] A. Zygmund, On a theorem of Marcinkiewicz concerning the interpolation of operations, J. de Math., 35 (1956), 223-248. 